\section{Kann Asthma ansteckend sein?}

\section{Im Spätsommer kommt es in Kanada jedes Jahr zu epidemieartig auf- tretenden Asthmanotfällen. Getriggert werden diese Exazerbationen vor allem durch virale Atemwegsinfektionen. In einer Studie sollte nach den verantwortlichen „Krankheitsvektoren“ gesucht werden.}

ro orscher der Universität Hamilton im kanadischen Bundesstaat Ontario erfragten vom Canadian Institute for Health Information die Daten aller Krankenhauseinweisungen der Jahre 1990 bis 2002. Dann suchten sie in deskriptiven Analysen nach geographischen Mustern der herbstlichen „Asthmaepidemien“, um daraus Vorhersagen über das zeitliche Auftreten bei Schulkindern, Vorschulkindern und Erwachsenen jeweils in Relation zum Schulbeginn nach den Sommerferien abzuleiten.

Der Höhepunkt der Asthmanotfälle trat bei Schulkindern jedes Jahr im Durchschnitt 17,7 Tage nach dem Labor Day auf. Dieser Feiertag fällt jeweils auf den ersten Montag im September und markiert das Ende der Sommerferien. Eine vergleichbare Häufung, wenn auch

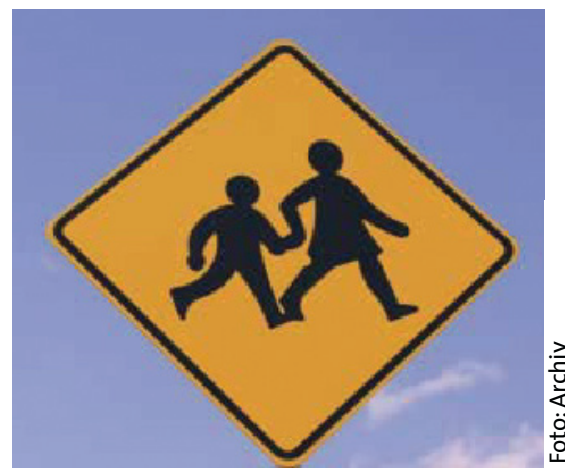

Achtung Asthmavektoren!

von geringerer Ausprägung, wurde 1,7 Tage später bei Vorschulkindern beobachtet. Bei Erwachsen schließlich war das Maximum der Zahl der Asthmaexazerbationen gegenüber den Schulkindern um 6,3 Tage verzögert. In Nordkanada traten zudem die Höhepunkte bei den Schulkindern 4,2 Tage früher auf als an der Grenze zu den USA.

Als Erklärung für ihre Beobachtungen vermuten die Forscher, dass die in die Schule zurückkehrenden Kinder als Vektoren der Asthmaexazerbation fungieren und die Auslöser verzögert an die Erwachsenen weitergeben. Wichtigster Triggerfaktor sind wohl Rhinovirusinfektionen, aber auch ein verändertes Allergenspektrum $-z$. B. aufgrund von Katzenallergentransport durch Mitschüler oder erhöhter Schimmelpilzbelastung in den Schulen - könnte eine Rolle spielen.

Fazit: Die in Kanada regelmäßig im September gehäuft auftretenden Asthmanotfälle stehen in enger zeitlicher Korrelation zum Schulbeginn nach den Sommerferien. Die Auslöser der Exazerbationen werden offenbar innerhalb der Schulklasse weitergegeben und von dort nach Hause getragen.

Johnston NW et al. The September epidemic of asthma hospitalization: school children as disease vectors. J Allergy Clin Immunol 2006; 117: 557-62

\title{
Rascher Erfolg bei Milbenasthma
}

\section{Modifizierte Allergene sollen die Verträglichkeit der Hyposensibili- sierung verbessern. In einer Studie wurden Wirksamkeit und Sicher- heit eines depigmentierten Allergenextrakts von Dermatophagoides pteronyssinus bei Kindern mit Milben-bedingtem Asthma untersucht.}

S panische Kinder im Alter zwischen acht und sechzehn Jahren mit leichtem bis moderatem Asthma aufgrund einer Monosensibilisierung gegen Dermatophagoides pteronyssinus erhielten über vier Monate entweder einen depigmentierten, polymerisierten $D$.-pteronyssinus-Extrakt $(\mathrm{n}=15)$ oder lediglich eine symptomatische Behandlung $(\mathrm{n}=15)$. Die Auftitration erfolgte mit vier Injektionen an zwei Tagen, gefolgt von der Erhaltungstherapie mit je einer Injektion im Monat. Die kumulative Dosis der insgesamt acht Injektionen in vier Monaten betrug $216,75 \mu \mathrm{g}$ des modifizierten Allergenextrakts.
Alle Patienten der Hyposensibilisierungsgruppe und 13 von 15 Patienten der Kontrollgruppe beendeten die Studie. Unter der Immuntherapie verbesserte sich im spezifischen bronchialen Provokationstest das $\mathrm{PC}_{20} \mathrm{FEV}_{1}$ gegenüber dem Ausgangswert signifikant $(\mathrm{p}<0,01)$. Die durchschnittliche Menge an nativem D.-pteronyssinus-Extrakt, die zur Auslösung eines 20\%igen Abfalls des $F E V_{1}$ erforderlich war, stieg in der aktiv behandelten Gruppe von $26 \mu \mathrm{g}$ zu Behandlungsbeginn auf $309 \mu \mathrm{g}$ am Ende, zwei Patienten zeigten nach vier Monaten gar keine Reaktion mehr. Bei den Kindern ohne Hyposensibilisierung dagegen blieb die Reaktion auf das Allergen konstant. Nur in der Immuntherapiegruppe war außerdem nach vier Monaten eine signifikante Reduktion der durch Pricktest bestimmten Hautreaktivität zu beobachten $(\mathrm{p}=0,0164)$. Auch bei den Symptom- und Medikationsscores waren die hyposensibilisierten Kinder im Vorteil.

Fazit: Eine viermonatige Hyposensibilisierung mit einem depigmentierten Allergenextrakt aus $D$. pteronyssinus führte bei Kindern mit Milben-bedingtem Asthma zu einer signifikanten Reduktion der bronchialen allergenspezifischen Reaktivität. Die Auftitration mit vier Injektionen an zwei Tagen war sicher. $\quad b k$

Ibero $\mathbf{M}$ et al. Significant improvement of specific bronchial hyperreactivity in asthmatic children after 4 months of treatment with a modified extract of Dermatophagoides pteronyssinus. J Investig Allergol Clin Immunol 2006; 16: 194-202 